Mathematical Modelling and Analysis

Volume 19 Number 5, November 2014, 676-695

http://dx.doi.org/10.3846/13926292.2014.980865

(c) Vilnius Gediminas Technical University, 2014
Publisher: Taylor\&Francis and VGTU

http://www.tandfonline.com/TMMA

Print ISSN: 1392-6292

Online ISSN: 1648-3510

\title{
Modeling Calcium Dynamics and Inhibition Based Synaptic Plasticity in Dendritic Spines
}

\section{Muhammad Dur-e-Ahmad}

\author{
Department of Applied Mathematics, University of Waterloo \\ ON, Canada \\ E-mail: mdureahm@asu.edu
}

Received March 12, 2014; revised September 25, 2014; published online November 1, 2014

\begin{abstract}
Inhibitory synapses through GABAergic channels not only change spine's calcium dynamics but also modulate postsynaptic current mediated through glutamate receptors. However the mechanism and extent to which these inhibitory synapses can modulate postsynaptic potential is not clearly understood. We propose a mathematical model to explain this phenomenon which encompasses both presynaptic and postsynaptic mechanisms. Further this model also elaborates the effect of these channels in synaptic calcium dynamics and learning mechanism.
\end{abstract}

Keywords: synaptic plasticity, calcium dynamics, NMDA, AMPA and GABAergic receptors, learning and memory.

AMS Subject Classification: $92 \mathrm{~B} 05$.

\section{Introduction}

One of the fundamental properties of nervous system is modifiable synaptic connections. These connections become strong during the developmental process, motivation as well as during the process of learning and memory and become weak when this process is slow $[1,3,16]$. The fundamental mode of these connections are synapses. These synapses are the primary mode of communications for neurons which allow them to form a network within the central nervous system. There are two types of synapses: electrical synapse and chemical synapse. Electrical synapse consists of a group of gap junctions occurring close together, while chemical synapse on the other hand, depends on the influx and efflux of specific types of proteins, called neurotransmitters. These neurotransmitters are enclosed in small membrane bound spheres called synaptic vesicles inside the presynaptic cells. When an action potential arrives at the presynaptic membrane, it produces an influx of calcium ions through voltage-dependent calcium channels. These calcium ions bind to these vesicles allowing them to fuse with the presynaptic membrane and release neurotransmitters into synaptic cleft through the fusion pores present on the membrane surface. These transmitters then move freely through the cleft and eventually arrive at the 
postsynaptic membrane. This chemical transmission in the brain is considered to be highly plastic because the postsynaptic potential can severely be affected due to the bi-directional movement of these transmitters in the region and their probable loss in synaptic cleft. $[22,24]$ (schematic of this activity is depicted in Figure 1). The strength of these synaptic connections can be modified as a result of activity at individual neuron. The terminal point of these transmitters are the receptors present on specialized protrusions on the dendritic surface of postsynaptic cell, called dendritic spines. These spines are femto-liter size protoplasmic protrusion and serve as the loci of synaptic plasticity $[13,29]$.

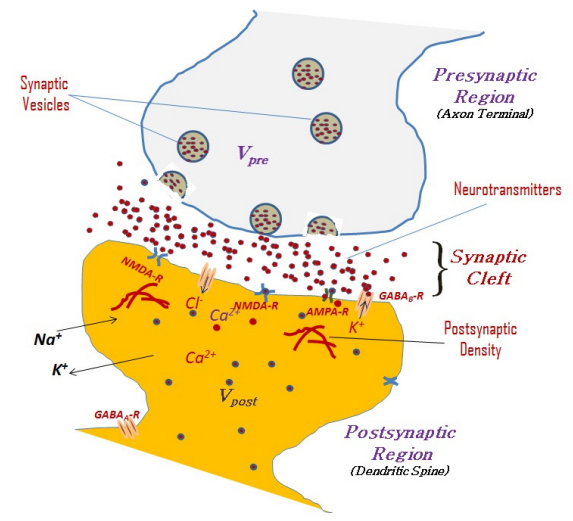

Figure 1. Pre and postsynaptic mechanism in neurons. Activity on the presynaptic terminal fuses synaptic vesicles and neurotransmitters are released into the synaptic cleft. These transmitters bind with the receptors on postsynaptic membrane and enter into the cytosol to change the transmembrane potential.

Spines are believed to restrict diffusion of ions. As such they form biochemical compartments that can encode changes in the state of an individual synapse without necessarily affecting the state of synapses of the same neuron $[12,25]$. The amount of neurotransmitters terminating on these spines depends on their concentration in the synaptic cleft. If the concentration of neurotransmitters is high they can frequently bind with the receptors located on spine's surface to increase the potential and open more voltage controlled ion channels. These neurotransmitters can both be excitatory, for example glutamate and inhibitory, for example GABAergic ( $\gamma$-Aminobutyric acid) [4,5,10,23]. Calcium entry into spines is greatly enhanced when an action potential is paired with synaptic stimulation and this enhancement requires the activation of voltage sensitive calcium channels such as N-methyl-D-aspartate (NMDA) receptor and calcium permeable $A M P A$ ( $(\alpha$-Amino-3-hydroxy-5-methyl-4-isoxazolepropionic acid) receptor channels. Activation of $N M D A$ receptors results in the opening of an ion channel which is nonselective to cations $[11,21,32]$. This allows the flow of $\mathrm{Na}^{+}$and small amounts of $\mathrm{Ca}^{2+}$ ions into the spine and $\mathrm{K}^{+}$out of the spine. These ions not only pass through the NMDA receptor channels but also modulate the activity of these receptors [14,18,31]. Generally, NMDA receptors do not open directly because their pores are occluded at resting membrane potential by $\mathrm{Mg}^{2+}$ ions $[10,20]$. NMDA receptors can only open when a depo- 
larization leads to repulsion of the $\mathrm{Mg}^{2+}$ cation out into the extracellular space, allowing the pores to pass current [32]. During the excitatory synaptic transmission, synaptic $N M D A$ receptors open to elevate calcium in the activated spines and eventually trigger the induction of long term potentiation (LTP) or long term depression (LTD) of synaptic transmission, a mechanism believed to be the fundamental basis of learning and the formation of memories in brain.

On the other hand inhibitory neurotransmitters activate receptors $G A B A_{A}$ and $G A B A_{B}$. Receptors $G A B A_{A}$ are found on dendritic spine heads and necks [5]. They are fast acting receptors that deal with chloride ions. $G A B A_{B}^{\prime} s$ receptors are slower in response as compared to $G A B A_{A}$ and are generally found near the dendritic shafts $[2,25]$. These receptors play a fundamental role in inhibition of synaptic activity by inhibiting presynaptic neurotransmitter release or dampening postsynaptic excitability state [9]. The role of $G A B A_{A}$ in postsynaptic cell is quite paradoxical. It has been observed in some cases that their activation in the early stage of synaptic activity can also contribute to excitation instead of inhibition [5]. But once these synapses are mature, they strongly inhibit the excitatory postsynaptic potentials $(E P S P)[5]$. In the mature stage of these synaptic activity these synapses play a direct role in inhibiting the action potentials. This inhibition is necessary in certain cases such as the situation where excessive excitatory synapse leads to seizures. Further they also create a balance of excitation and inhibition which is crucial to the normal functioning of all neurons. Excess of excitation or inhibition may lead the cells to enter a hyperexcitable (epileptiform) or silent (comatose) state [9]. Therefore $G A B A$-R's can potentially become part of many therapeutic drugs to prevent mental and physical disorders in the human body.

During recent years many studies and models have come up talking about $A M P A$ and $N M D A$ receptors, see for instance [10,14,32], but still there has been a surge in interest in $G A B A$ receptors due to their significant contribution to synaptic plasticity. Further, the role of $G A B A$ in synaptic modulation in the postsynaptic cell is still unclear. Recently, it has been observed, that $G A B A$ receptors not only shorten the action potential after depolarization and generate inhibitory postsynaptic potentials but also strongly inhibit NMDA dependent calcium signals $[4,5]$. However, the extent to which this modulation of Calcium signals occurs during the activation of $G A B A$ receptors in spines remains unknown. Furthermore research and investigations are required to understand this complete mechanism of synaptic modulations in spine in the presence of active $G A B A$ receptors.

Here we propose a mathematical model to understand the role of $G A B A$ in the plasticity of calcium signals evoked on dendritic spine. These models cover the scenarios of pre and postsynaptic mechanism in the cells and explain how the spine calcium transmitted through glutamate receptors is modulated in the presence of active inhibitory receptors. The model we have proposed is fundamentally probabilistic because not all of the neurotransmitters leaving presynaptic neuron arrive the adjacent postsynaptic membrane. Some are lost in the synaptic cleft due to various reasons including the level of concentration of ions, reactions with other ions in the cleft as well as diffusion out of the cleft. So we calculate the conditional probability that a neurotransmit- 
ter molecule leaving the presynaptic cell will reach a receptor on postsynaptic cell and include that element of probability in our entire pre and postsynaptic models.

This paper has three main part. In the first section, we develop a model to explain a mechanism of neurotransmitter release from the presynaptic cell caused by the external activities. The second part explains the detailed dynamics of postsynaptic mechanism in the cell. In this part we discuss how the action potential induced by $N M D A$ and $A M P A$ channels are modulated in the presence of active $G A B A$ channels. Further the plasticity of calcium signals induced through $N M D A$ and $A M P A$ in the presence of $G A B A$ inhibitory signals is also discussed. To avoid the complexity, we only discuss the case when $G A B A_{A}$ contribute towards synaptic inhibition. In the final part of the paper, we discuss how the synaptic strength between the coupled pre and postsynaptic cells is affected due to the modulation of calcium signals and define a LTP based learning model.

\section{Dynamic Model of Plasticity}

The synaptic plasticity model is divided into two compartments, the presynaptic region and postsynaptic region (see Figure 1 for schematic description). Although both of these compartments are not physically connected but they communicate with each other via chemical synapses. These chemical synapses are formed due to the release of neurotransmitters from the presynaptic cell and after traveling through synaptic cleft, are terminated on the postsynaptic surface, i.e., dendritic spines. We will discuss the mathematical formulation of these regions separately in the following subsections.

\subsection{Presynaptic dynamics}

In order to model the activity on the presynaptic terminal, we assume that it is an isopotential compartment and let $V_{\text {pre }}(t)$ denote the membrane potential of the presynaptic cell. The total capacitance on the terminal is calculated by the relation $C_{\text {pre }}=A_{\text {pre }} C_{m}(\mu F)$ where $A_{\text {pre }}$ is the presynaptic terminal surface area and $C_{m}$ is specific membrane capacitance. When an action potential moves through a presynaptic region, It will increase the transmembrane potential in the cell. The current balance equation for the membrane potential in a presynaptic terminal is given by

$$
C_{\text {pre }} \frac{d V_{\text {pre }}}{d t}=-I_{\text {ion }}-I_{\text {syn }}
$$

Here $I_{i o n}$ represents ionic current passing through the terminal and $I_{\text {syn }}$ represents the synaptic current. The ionic current is the current that flows due to the movement of ions through the ionic channels located on the cell membrane. For the excitable membrane, we used the similar mechanism as used in [10] based on Hodgkin-Huxley kinetics. The equation for the ionic current is given 
by

$$
I_{\text {ion }}=A\left[\left(V_{\text {pre }}-V_{N_{a}}\right) \bar{g}_{N_{a}} m^{3} h+\left(V_{\text {pre }}-V_{K}\right) \bar{g}_{K} n^{4}+\left(V_{\text {pre }}-V_{L}\right) \bar{g}_{L}\right] .
$$

Here $A$ is the surface area of presynaptic terminal, $V_{N a}, V_{K}$ and $V_{L}$ are the reversal potentials for the sodium, potassium and leakage currents with maximal conductances $\bar{g}_{N_{a}}, \bar{g}_{K}$ and $\bar{g}_{L}$ respectively. The activation and inactivation variables $m, n$ and $h$ satisfy the standard first order equations given by

$$
\begin{aligned}
& \frac{d m}{d t}=\alpha_{m}(1-m)-\beta_{m} m, \\
& \frac{d n}{d t}=\alpha_{n}(1-n)-\beta_{n} n, \\
& \frac{d h}{d t}=\alpha_{h}(1-h)-\beta_{h} h,
\end{aligned}
$$

where

$$
\begin{array}{ll}
\alpha_{n}=\frac{0.01 \phi\left(10-V_{\text {pre }}\right)}{\exp \left(\frac{10-V_{\text {pre }}}{10}\right)-1}, & \beta_{n}=0.125 \phi \exp \left(-\frac{V_{\text {pre }}}{80}\right), \\
\alpha_{m}=\frac{0.1 \phi\left(25-V_{\text {pre }}\right)}{\exp \left(\frac{25-V_{\text {pre }}}{10}\right)-1}, \quad \beta_{m}=4 \phi \exp \left(-\frac{V_{\text {pre }}}{18}\right), \\
\alpha_{h}=0.07 \phi \exp \left(-\frac{V_{\text {pre }}}{20}\right), \quad \beta_{h}=\frac{\phi}{\exp \left(\frac{30-V_{\text {pre }}}{10}\right)+1} .
\end{array}
$$

Here $\phi=3^{\frac{T-3.3}{10}}$ where $T$ is the temperature which is assumed to be $22^{\circ} \mathrm{C}$.

In our model we are taking into account the postsynaptic conductance of the previous neuron as well which becomes the input signal for our presynaptic neuron. For the sake of simplicity, we used $\alpha$-function to model the synaptic activity on this region, $[6,30]$. So, in this case the synaptic current $I_{\text {syn }}$ is calculated as follows:

$$
I_{\text {syn }}=g_{\text {syn }}\left(V_{\text {pre }}-V_{\text {syn }}\right),
$$

where $V_{\text {syn }}$ is the synaptic reversal potential and $g_{\text {syn }}$ is a brief synaptic conductance and is modeled by using periodic function

$$
\begin{aligned}
& g_{\text {syn }}(t)= \begin{cases}g_{p} \frac{t}{t_{p}} e^{1-\frac{t}{t_{p}}}, & t \in\left[0, t_{p}\right], \\
0, & \text { otherwise, }\end{cases} \\
& g_{\text {syn }}\left(t+k t_{p}\right)=g_{\text {syn }}(t), \quad k=1,2, \ldots
\end{aligned}
$$

This equation can be solved to calculate the change in presynaptic potential versus time as a result of synaptic firing and ionic currents. $V_{\text {pre }}$ is a function of time. It rises and falls with each action potential caused by the preceding chemical synapse.

Before solving the model in detail a few other factors need to be considered. It is important to state that we have modeled each neuron as a single compartment. Further the release of neurotransmitter from presynsptic cell can be spontaneous or activity dependent as discussed in $[7,17]$. The mechanism we 
adopted here is stochastic in the sense that it uses the average probabilities of neurotransmitter release from all sites regardless of their nature. Since the probability of the neurotransmitter release is enhanced in the presence of activity, therefore when an action potential arrives on the presynaptic terminal, it causes the vesicles filled with neurotransmitters to fuse out of the cell into the synaptic cleft along the membrane surface. The concentration of neurotransmitter in the synaptic cleft depends directly on the presynaptic potential [7]. We will use a continuous function to transform the presynaptic voltage into transmitter concentration $[\mathrm{T}]$. In this approach we assume that all intervening reactions in the release process are relatively fast and can be considered in steady state. The stationary relationship between the transmitter concentration and the presynaptic voltage is fit to

$$
[T]\left(V_{\text {pre }}\right)=\frac{T_{\max }}{1+\exp \left[-\left(V_{\text {pre }}-V_{p}\right) / K_{p}\right]},
$$

where $T_{\max }$ is maximal concentration of transmitter in the synaptic cleft and is usually taken to be $1 \mathrm{mM}, K_{p}=5 \mathrm{mV}$ gives the maximum threshold of transmitters release and $V_{p}=2 m V$ represents the half activated threshold release.

These neurotransmitters can have an effect on the postsynaptic neuron by binding with either to directly activated receptor channels or by binding with a distinct receptor that activates the synaptic conductance indirectly through an intracellular signalling pathway [7]. In the case of the directly activated receptor channels, the transmitters react with the channels. In the forward reaction the transmitter molecules bind to a receptor and open it. In the backward reaction these transmitters may also unbind from the receptor which will eventually close it. This opening and closing results in varying open probability for the channel with time. We denote this probability by $P_{s}$ and it can be modeled as follows:

$$
\frac{d P_{s}}{d t}=\alpha_{s}\left(1-P_{s}\right)-\beta_{s} P_{s}
$$

Here $\beta_{s}$ is the closing rate of the channel and is taken to be $190 s^{-1}, \alpha_{s}$ is the opening rate of the channel which depends on the transmitter concentration and $[T]$ is concentration of neurotransmitters in the synaptic cleft. Assuming that $k$ transmitter molecules are required to bind with a channel, then the probability of $k$ transmitter molecules being within the binding range of the receptor is proportional to $[T]^{k}$ and $\alpha_{s}=r[T]^{k}$, where $r$ is the constant of proportionality. So the above equation can be written as

$$
\frac{d P_{s}}{d t}=r[T]^{k}\left(1-P_{s}\right)-\beta_{s} P_{s}
$$

The value of $P_{s}$, calculated from equation (2.1), will be used in the models of postsynaptic region.

\subsection{Postsynaptic dynamics}

When an action potential from the presynaptic region arrives on the postsynaptic cell, it will open the receptors on the postsynaptic membrane which will 
allow bi-directional conduction of ionic flux through them. The postsynaptic cell will depolarize or hyperpolarize due to the ion exchange across the excitatory or inhibitory receptors respectively. In defining a postsynaptic model, it is important to consider the type of receptors that are involved due to their different synaptic activities. The primary receptors we will be considering for our paper are $A M P A, N M D A, G A B A_{A}$ and $G A B A_{B}$. AMPA and NMDA are glutamate type receptors and are responsible for the induction of $\mathrm{Ca}^{2+}$ along with some other cations which results in the rise of excitatory postsynaptic potential ( $E P S P$ ), whereas $G A B A$ 's are mainly responsible for the inhibitory postsynaptic potential (IPSP). Further, $N M D A, A M P A$ and $G A B A_{A}$ are ionotropic type receptors, which binds neurotransmitters to the synaptic channel and activates it directly, while the $G A B A_{B}$ are metabotropic in nature in which the transmitter binds to a distinct receptor that activates the channel indirectly through an intracellular signalling pathway. As a whole, we can write the current balance equation for the membrane potential in postsynaptic spine as

$$
C_{\text {post }} \frac{d V_{\text {post }}}{d t}=-I_{\text {ion }}-I_{X}-I_{G A B A_{B}},
$$

where $I_{i o n}$ is Hodgkin-Huxley type ionic current, as we have in the presynaptic region while $I_{X}$ is synaptic current through ionic channels. The postsynaptic current occurs as a result of rise and fall in potential caused by the firing of the presynaptic neuron. So a general model for postsynaptic current $I_{X}$ can be written mathematically as follows:

$$
I_{X}=g_{X} P_{s}\left(V_{\text {post }}-V_{X}\right),
$$

where $V_{X}$ is the reversal potential of the postsynaptic channel, $P_{s}$ is the probability that the channel is in open state and $g_{X}$ is channel conductance. The subscript " $X$ " represents $A M P A, N M D A$ or $G A B A_{A}$. In the current balance equation (2.2), we separate $G A B A_{B}$ current since its nature is slightly different than the remaining three synaptic currents due to its metabotropic nature. We will discuss its formation later in this section.

Since in this paper, we are dealing with two different types of synaptic activities, excitatory and inhibitory, therefore, we can break down $I_{X}$ as follows:

$$
I_{X}=I_{e x}+I_{G A B A_{A}} .
$$

Further, since the excitatory current $I_{e x}$ consists of $I_{A M P A}$ and $I_{N M D A}$, thus the above equation can be written in the expanded form as

$$
I_{X}=I_{A M P A}+I_{N M D A}+I_{G A B A_{A}} .
$$

The models for synaptic conductances are discussed below.

NMDA-Conductance. The NMDA receptor channels are a major source of calcium entry into spine which are believed to play a key role in the summation of synaptic responses and the generation of synaptic plasticity. They are gated by magnesium in a voltage dependent manner and are highly permeable to calcium. When a synapse is activated by high frequency stimulation, 
it depolarize the postsynaptic membrane to remove the magnesium block of $N M D A$ receptors and as a result calcium enters through the open $N M D A$ receptors $[10,32]$. The $N M D A$ conductance $g_{N M D A}$ is modeled as a function of both time and postsynaptic potential and is formulated in such a way that the binding rate constant for binding magnesium to the site of the channel block varies as a function of membrane voltage. Specifically,

$$
g_{N M D A}(t)=\bar{g}_{N M D A} \frac{e^{-t / \tau_{1}}-e^{-t / \tau_{2}}}{1+\eta\left[M g^{2+}\right] e^{-\xi V_{p o s t}}} .
$$

Here $\bar{g}_{N M D A}$ is the maximum conductance of the NMDA receptors and its value is $30 \mathrm{pS}$. $\tau_{1}$ and $\tau_{2}$ are the time constants for synaptic conductance decay and rise and their values are $139 \mathrm{~ms}$ and $0.6 \mathrm{~ms}$ respectively, $\eta$ and $\xi$ are constants expressing the voltage dependent blocks by $\mathrm{Mg}^{2+}$ and their values are 0.33 per $m M$ and 0.06 per $m V$ respectively [10].

AMPA-Conductance. The channels for the $A M P A$ current are also activated in a similar fashion as $N M D A$ channels with a periodic simulation of a cluster of synapses [10]. The expression for $A M P A$ conductance $g_{A M P A}$ is given by

$$
g_{A M P A}(t)=\bar{g}_{A M P A}\left(e^{-t / T_{d}}-e^{-t / T_{r}}\right) .
$$

Here $\bar{g}_{A M P A}$ is the maximum conductance of the $A M P A$ receptors and is set at $10 p S, T_{d}$ and $T_{r}$ are the decay and rising time constants with the values of 1 $m s$ and $0.4 \mathrm{~ms}$ respectively.

GABA $_{A}$-Conductance. $G A B A_{A}$ is the third ionotropic channel on the postsynaptic membrane along with the first two excitatory receptors. These are fast-responding $G A B A$ receptors and are modeled in a similar periodic way as the first two ionotropic receptors $N M D A$ and $A M P A$. The model for $g_{G A B A A}$ is given by

$$
g_{G A B A_{A}}(t)=\bar{g}_{G A B A_{A}} f\left(e^{-t / T_{\text {decay }}}-e^{-t / T_{\text {rise }}}\right) .
$$

Here $f$ is the normalization factor and is included to ensure that amplitude equals $\bar{g}_{G A B A}$, the maximum peak conductance, and is given by the relation

$$
f=\frac{1}{-e^{-t_{\text {peak }} / T_{\text {rise }}}+e^{-t_{\text {peak }} / T_{\text {decay }}}}, \quad t_{\text {peak }}=\frac{T_{\text {decay }} T_{\text {rise }}}{T_{\text {decay }}-T_{\text {rise }}} \ln \left(\frac{T_{\text {decay }}}{T_{\text {rise }}}\right) .
$$

The value of $\bar{g}_{G A B A A}$ is chosen to be $1.2 n S$ [28]. The values of rise and decay time constants are $2.5 \mathrm{~ms}$ and $1.3 \mathrm{~ms}$ respectively.

$G A B A_{B}$-Current. $G A B A_{B}$ is the only metabotropic receptors amongst the four that we have discussed in the paper. Therefore the model for $G A B A_{B}$ 
does not involve probability but it depends on a direct coupling of an intracellular "second messenger" that is produced when a neurotransmitter binds to a separate receptor molecule. This response is mediated by $K^{+}$channels that are activated by G-protein. The mathematical formulation of $G A B A_{B}$ current is as follows.

$$
I_{G A B A_{B}}=g_{G A B A_{B}} \times \frac{g^{n}}{g^{n}+k_{d}}\left(V_{\text {post }}-V_{G A B A_{B}}\right),
$$

where $V_{G A B A_{B}}$ is reversal potential and $g_{G A B A_{B}}$ is $G A B A_{B}$ conductance. We are using the same expression for $g_{G A B A_{B}}$ as of $g_{G A B A_{A}}$ except the decay and rise time constant are $120 \mathrm{mS}$ and $5 \mathrm{mS}$ respectively. $r$ is the concentration of activated receptors and $g$ is the concentration of activated second messenger(G-protein). These are calculated from the following equations

$$
\begin{aligned}
& \frac{d g}{d t}=k_{3} r-k_{4} g, \\
& \frac{d r}{d t}=k_{1}[T](1-r)-k_{2} r,
\end{aligned}
$$

where $\bar{g}_{G A B A_{B}}=1 n S$. The parameter values we are using are $n=4, k_{d}=$ $100 \mu M^{4}, k_{1}=9 \times 10^{4} M^{-1} s^{-1}, k_{2}=1.2 s^{-1}, k_{3}=180 s^{-1}, k_{4}=34 s^{-1}$ [28].

\subsection{Modeling calcium dynamics}

Calcium is one of the most important intracellular component which is responsible for the intracellular triggering of hormone secretion. They are believed to be the integral part of exocytotic pathways due to their spatio-temporal patterns in cytosol generated by the subtle interplay between intracellular calcium source and its removal. Besides this, calcium signals invoked through NMDA channels are consider to be the key player behind LTP and LTD, a neurocellular basis of learning and memory formed in hippocampus [19,32]. Recently it has been observed that the calcium signals in the postsynaptic cells are strongly modulated under the influence of GABAergic inhibitory effects $[4,5]$. But due to the intracellular complexities, the extent of this modulation is still unknown. In order to understand this effect, we develop the following calcium dynamic model which incorporate both excitatory and inhibitory effects

$$
\frac{d[C a]}{d t}=\epsilon_{0}\left(C a-C_{m i n}\right)+K_{1}\left|I_{e x}-I_{i n h}\right|,
$$

where $\epsilon_{0}$ is a rate constant, $C_{\text {min }}$ is the minimum store of calcium in the postsynaptic cell and $K_{1}$ is the binary constant that determines the rate how the excitatory or inhibitory currents are affecting the calcium concentration. The above equation measures the rate of change of calcium concentration with time in response to the modulations of excitatory and inhibitory synapses.

\subsection{Learning rule}

The mechanism of learning in the brain is widely believed to be associated to the synaptic plasticity. This means if we have strong connection between 
neurons, learning will be enhanced otherwise it will be weak $[15,19]$. Since neuronal calcium is the key player behind many forms of synaptic modulations, therefore, it also plays an important role in the formation of new memories and learning mechanism. According to the calcium control hypothesis, basal levels of calcium concentration do not lead to any changes in synaptic efficacy but modest and high levels of calcium concentration lead to LTP and excitotoxity stage respectively [29].

Based on these calcium hypothesis, we use the following model to explain the learning mechanism in neurons.

$$
\tau_{w}([C a]) \frac{d w}{d t}=\epsilon_{1}\left(\Omega_{w}([C a]-w)\right) .
$$

Observe that here the synaptic weight $w$ is a function of intracellular calcium. Further, $\epsilon_{1}$ is rate constant and $\tau_{w}([\mathrm{Ca}])$ is the calcium dependent learning time constant which is inversely related to the learning rate $\eta_{w}$, i.e.,

$$
\tau_{w}([C a])=\frac{1}{\eta_{w}} .
$$

This learning rate $\eta_{w}$ is related to neuronal calcium concentration through the relation

$$
\eta_{w}=A+[C a]^{B}
$$

where $A$ and $B$ are constant and we take their values to be $1 \mathrm{~ms}$ and $3 \mathrm{~ms}$ respectively. $\Omega_{w}([\mathrm{Ca}])$ is also a function of calcium concentration and is modeled as a difference of two sigmoids as follows

$$
\Omega_{w}([C a])=0.25+\frac{1}{1+\exp \left(-\beta_{2}\left([C a]-\alpha_{2}\right)\right)}-\frac{0.25}{1+\exp \left(-\beta_{1}\left([C a]-\alpha_{1}\right)\right)}
$$

with $\alpha_{1}=0.25, \alpha_{2}=0.55, \beta_{1}=80, \beta_{2}=80$. Using these framework of learning, we analyzed the direction and strength of calcium dependent plasticity and the results are discussed in the following section.

All the parameter values we used in our model along with their description are listed in Table 1.

\section{Simulation Results}

The equations in our model are all connected to each other and so had to be solved as a system of coupled differential equations. The system is integrated using stiff solver ODE15s, with maximum step size of $1 \mathrm{~ms}$, from the MATLAB ODE suite. ODE15s is an implicit Runga Kutta method based ODE routine in MATLAB. The results of each variable were stored in a vector and plotted with respect to time to derive the significance.

Activity Dependent Neurotransmitter release from the Presynaptic cell: When an action potential invades on the presynaptic cell, it depolarizes the surface and excitatory presynaptic potential rises. As a result, the synaptic vesicles inside the membrane break to release the neurotransmitters. These 
Table 1. Parameter values for the model.

\begin{tabular}{|c|c|c|}
\hline Symbol & Parameter value & Description \\
\hline$A$ & $1 \cdot 10^{-8} \mathrm{~cm}^{2}$ & Surface area of the spine head \\
\hline$\beta_{S}$ & $190 S^{-1}$ & Closing rate of postsynaptic channel \\
\hline$C_{\text {post }}$ & $10^{-5} \mathrm{mF} / \mathrm{mm}^{2}$ & Specific membrane capacitance \\
\hline$C_{\text {pre }}$ & $10^{-5} \mathrm{mF} / \mathrm{mm}^{2}$ & Specific membrane capacitance of presynaptic cell \\
\hline$\epsilon_{0}$ & $0.02 N A$ & Rate constant \\
\hline$\epsilon_{1}$ & $1 \times 10^{-4} N A$ & Scaling constant for learning equation \\
\hline$\eta$ & $0.33 \times 10^{-6} \mathrm{~V}$ & Constant in the $N M D A$ conductance model \\
\hline $\bar{g}_{K}$ & $0.36 \mathrm{~ms} / \mathrm{mm}^{2}$ & Maximal potassium conductance \\
\hline $\bar{g}_{L}$ & $0.003 \mathrm{~ms} / \mathrm{mm}^{2}$ & Maximal leakage conductance \\
\hline $\bar{g}_{N a}$ & $1.2 \mathrm{~ms} / \mathrm{mm}^{2}$ & Maximal sodium conductance \\
\hline$g_{p}$ & $7.4 \cdot 10^{-11} S$ & Peak synaptic conductance in presynaptic cell \\
\hline$g_{A M P A}$ & $10^{-12} S$ & AMPA peak Conductance \\
\hline$g_{G A B A_{A}}$ & $1.2 \times 10^{-9} \mathrm{mV}$ & $G A B A_{A}$ peak Conductance \\
\hline$g_{G A B A_{B}}$ & $10^{-9} \mathrm{mV}$ & $G A B A_{B}$ peak Conductance \\
\hline$g_{N M D A}$ & $30^{-12} \mathrm{mV}$ & NMDA eak Conductance \\
\hline$K_{1}$ & $1 \times 10^{8} N A$ & Control variable \\
\hline$K_{p}$ & $5 m V$ & Stiffness of release \\
\hline$M g$ & $1.2 \times 10^{6} n M$ & Concentration of magnesium ions \\
\hline$V_{K}$ & $-77 m V$ & Potassium reversal potential \\
\hline$V_{L}$ & $-54.387 m V$ & Leakage reversal potential \\
\hline$V_{N a}$ & $50 m V$ & Sodium reversal potential \\
\hline$V_{A M P A}$ & $60 m V$ & AMPA reversal potential \\
\hline$V_{G A B A}^{A N F A}$ & $-70 m V$ & $G A B A_{A}$ reversal potential \\
\hline$V_{G A B A_{B}}$ & $-95 m V$ & $G A B A_{B}$ reversal potential \\
\hline$V_{N M D A}$ & $60 m V$ & NMDA reversal potential \\
\hline$V_{\text {syn }}^{N M D A}$ & $10^{2} \mathrm{mV}$ & Synaptic reversal potential \\
\hline$V_{p}$ & $2 m V$ & Threshold of release \\
\hline$T_{\max }$ & $1 m M$ & Maximum neurotransmitter concentration \\
\hline$t_{p}$ & $0.2 \mathrm{~ms}$ & Peak conductance time \\
\hline
\end{tabular}

transmitters then diffuse into the synaptic cleft through specialized channels present on the cell membrane.

For the simulation purpose, we used three different level of activities to increase the intensity of action potential, (a) the $\alpha$-range of $10 \mathrm{~Hz}$ (Figure 2A), (b) medium $\gamma$-range of $40 \mathrm{~Hz}$ (Figure 2B) and (c) high $\gamma$-range of $80 \mathrm{~Hz}$ (Figure $2 \mathrm{C}$ ) to depolarize the presynaptic membrane. Other than the frequency changes, all other conditions are kept the same. The simulations were run in a time window of $500 \mathrm{~ms}$. It is clear from the results that the potential rises from $0 \mathrm{mV}$ to about $90 \mathrm{mV}$ in all three cases which exhibit excitatory action potential. This activity is directly related to the release of neurotransmitters from the presynaptic cell. Higher activity in the presynaptic cell corresponds to the higher release of neurotransmitters which will increase their probability to attach with a receptor on postsynaptic cell. Figure 2(D-F) represents the graphs of changing the open probability of receptors on postsynaptic cell. These graphs are also plotted for the same frequency ranges of $10 \mathrm{~Hz}, 40 \mathrm{~Hz}$ and $80 \mathrm{~Hz}$ to make a relation with the presynaptic activity. It can be seen that the probability graphs closely follow the spikes of the presynaptic potential graphs from Figure $2(\mathrm{~A}-\mathrm{C})$. This indicates that as the synaptic potential rises 

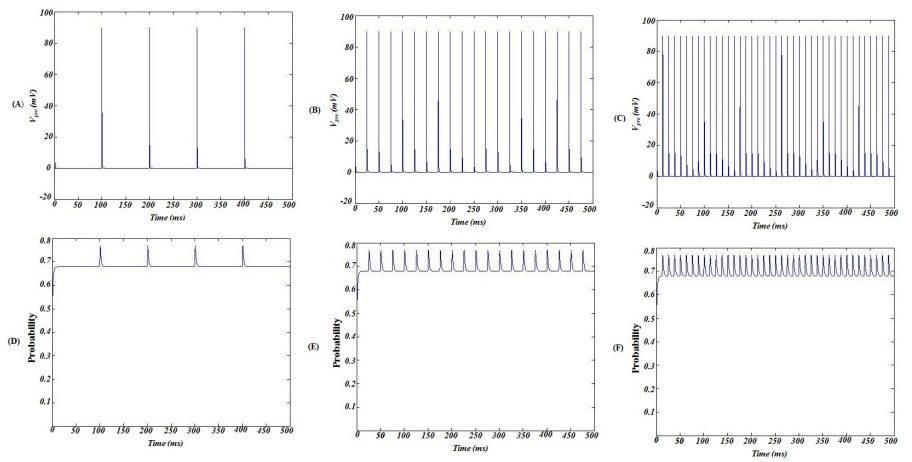

Figure 2. Top Panel: Change in transmembrane potential in presynaptic axonal terminal. Three different level of activities are used to depolarize the membrane.

Panel (A) $\alpha$-range of $10 \mathrm{~Hz}$, Panel (B) medium $\gamma$-range of $40 \mathrm{~Hz}$ and Panel (C) high $\gamma$-range of $80 \mathrm{~Hz}$. Bottom Panel:Strong activity in the presynaptic terminal will intensify the release of neurotransmitters in the cleft and therefore the probability of opening and closing of gates on the postsynaptic side will also increase.

and falls, the open probability of the channels on the postsynaptic cell also increases and decreases with the same frequency. In other words, more activity on the presynaptic cell corresponds to a frequent opening of channels on the postsynaptic cell and vice versa. The value of this probability remains between 0.6 to 0.8 at all times. Changing the activity in the presynaptic cell has an obvious effect on the number of spikes of probability but a very minute effect on its magnitude.
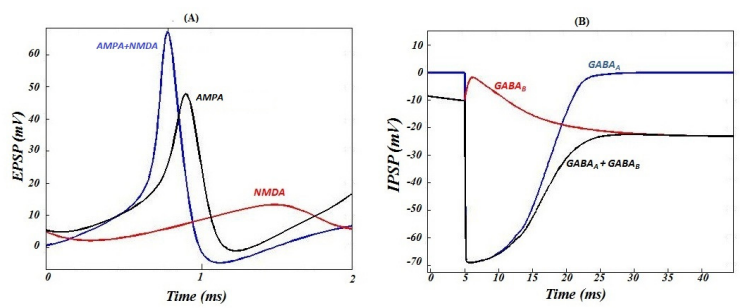

Figure 3. Comparison of spike characteristics of mediated currents in postsynaptic cell through different channels. Panel A: EPSP mediated by $A M P A$ and NMDA channels.

Panel B: IPSP mediated by $G A B A_{A}$ and $G A B A_{B}$ channels

Postsynaptic Dynamics: The first step is to obtain a description of inhibitory and excitatory synaptic mediated conductance and their contribution towards postsynaptic potential change. To observe this we use equation (2.2) and observe the change in the postsynaptic potential. The response of activation of these channels (in both of the cases, separately and simultaneously) is determined by using a test pulse for a duration of two $m s$ and the results are depicted in Figure 3. Although the relative amplitudes of $A M P A$ and NMDA 
receptor mediated components of EPSP have been found widely, the amplitude of the $N M D A$ receptor mediated component is usually smaller than that of $A M P A$ receptor mediated component (Figure $3 \mathrm{~A}$ ). This rapid time course of $A M P A$ response is thought to be due to the combination of rapid clearance of neurotransmitters and rapid channel closure and the blockage of these channels can cause a fast synaptic depression. Further, both NMDA and AMPA receptor channels show a significant facilitation to produce somatic EPSP during activation. When both channels are activated at the same time, the amplitude of the resulting EPSP is slightly higher than the EPSP spike generated by $A M P A$ alone. Similarly, the effect of a single spike of fast inhibitory synapse through $G A B A_{A}$ and slow inhibitory response through metabotropic receptor $G A B A_{B}$ is depicted in Figure 3B. These results are in accordance with the experimental data, see for instance $[8,26]$.
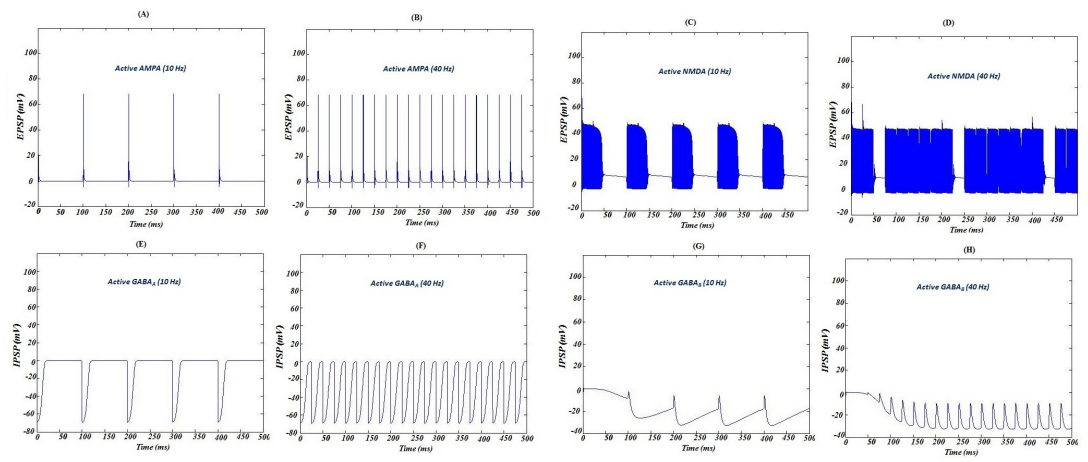

Figure 4. Top Panel: Postsynaptic activity due to excitation. (A and B) Activity due to the $A M P A$ mediated current and (C and $\mathbf{D})$ Activity due to $N M D A$ mediated current.

Bottom Panel:Postsynaptic activity due to inhibition. $\mathbf{E}$ and $\mathbf{F}$ Activity due to the ionotropic $G A B A_{A}$ channel and $\mathbf{G}$ and $\mathbf{H}$ Activity due to the active metabotropic $G A B A_{B}$ channels. Clearly the response of $G A B A_{B}$ currents is different than to $G A B A_{A}$ current as the later one requires a strong stimuli to activate due to its metabotropic nature.

Experiment is performed for two different level of mediated activities in all these cases,

(A, C, E and G) $10 \mathrm{~Hz},(\mathbf{B}, \mathbf{D}, \mathbf{F}$ and $\mathbf{H}) 40 \mathrm{~Hz}$.

In Figures 4, we have isolated the change in postsynaptic potential caused by the movement of ions through $A M P A$ and $N M D A$ receptors. In Figures 4(A and $\mathrm{B})$, all other channels except $A M P A$ are blocked and hence they are not contributing towards EPSP. It is clear that potential in both of the graphs is rising from zero to a positive value which represents that $A M P A$ is only responsible for excitatory synapse. Here simulations are run for two different frequency ranges, $10 \mathrm{~Hz}$ and $40 \mathrm{~Hz}$. Simulations for $80 \mathrm{~Hz}$ also follow the same pattern (not shown here). Similarly, in Figure 4(C and D), only NMDA channel is active and the effect of opening and closing of these receptors is shown. Since $N M D A$ receptors also contributes to EPSP, thus the higher activity will increase the density of spikes. Although the amplitude of $N M D A$ spikes are smaller than that of $A M P A$ but they are very dense and a clear periodic pattern can only be observed for the $10 \mathrm{~Hz}$ activity. This type of activity behavior 
through NMDA channels is very common and has been documented extensively in literature both via experiments and modeling work, see for example [10,27, $32,33]$.

Similar mechanism is adapted to isolate the inhibitory channels from the excitatory channels. Fast inhibitory postsynaptic potentials (IPSPs) are mediated by $G A B A_{A}$ receptors. Opening of these channels can generate a shunting conductance that will eventually change the passive properties of the membrane. It is evident from the Figure $4(\mathrm{E}-\mathrm{H})$ that the response of both of the inhibitory receptors are different and $G A B A_{A}$ mediated IPSP has relatively longer amplitude as compare to $G A B A_{B}$, which require a strong stimuli due to its metabotropic nature. The quick response of the $G A B A_{A}$ receptor is due to its high affinity for the inhibitory synapses and it is believed that they are saturated even by the release of a single vesicle of neurotransmitter [26]. Unlike the $G A B A_{A}$ receptors, responses from $G A B A_{B}$ require high level of presynaptic activity. This can be either due to the extrasynaptic localization of these receptors or due to the cooperativity in the activation kinetics of $G A B A_{B}$ response [8]. As a consequence, the dynamics of the $G A B A_{B}$ responses are very difficult to capture experimentally and therefore we need to rely on modeling.
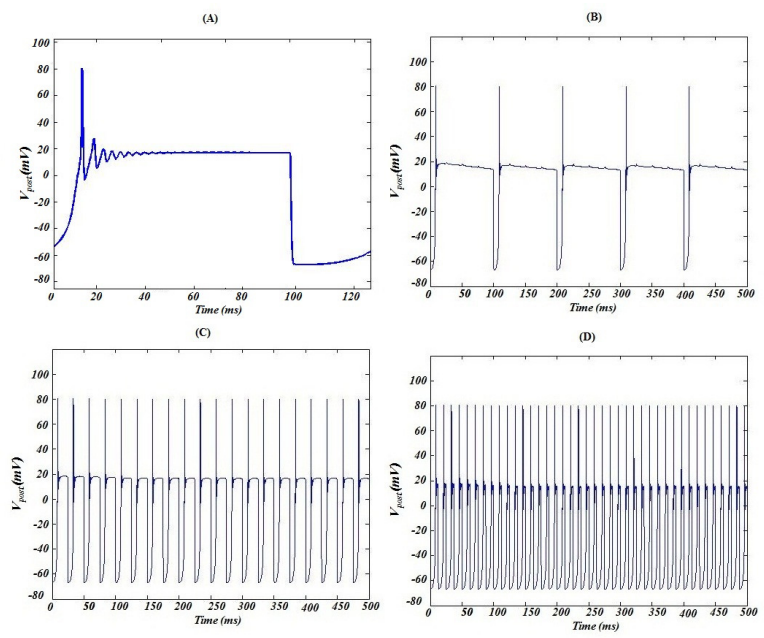

Figure 5. All of the four $A M P A, N M D A, G A B A_{A}$ and $G A B A_{B}$ channels are active and contributing in rise of postsynaptic potential. Strong excitatory and inhibitory effects are visible. Inhibition is quite strong to develop a sharp hyperpolarization and afterhyperpolarization. Panel (A): An isolated single spike shows the behavior when all four channels are active. Again the experiment is run for three different level of activities,

(Panel B:) 10Hz, (Panel C:) 40Hz and (Panel D:) $80 \mathrm{~Hz}$.

Finally, when all the four channels are active together, then we can see a complete picture of postsynaptic potential. A strong depolarization due to the active excitatory channels as well as sharp hyperpolartion and afterhyperpolarization due to the active inhibitory synapses is evident (Figure 5A). A sharp spike quickly arise in the beginning because the excitatory channels activate earlier than the GABAergic channels. But due to the strong inhibition, they 
are not only brought back to the baseline position but also forced to push further down and a very strong afterhyperpolarization can be seen. Also some spike fluctuation is also visible near the baseline when spike returns. This is due to the strong action of $N M D A$ channel which keep this activity alive before afterhyperpolarization. After the first spike, although neuron tries to fire another action potential due to this $N M D A$ channel activation, but it is not able to cross the firing threshold because of strong inhibitory effect. But once the inhibitory spike is complete this cycle will resume. In Figure 5(B-D), a complete picture of postsynaptic activity is shown in $500 \mathrm{~ms}$ window. All the results in these figures are obtained by keeping the same activity level for all of the four synapses. For example, Figure 5B shows the result when all of the channels are activated with $10 \mathrm{~Hz}$ frequency and so on.

Plasticity of Calcium signals and Learning Rule: Since calcium is one of the most important component behind many forms of synaptic plasticity and LTP induced learning and memory, therefore, it is important to analyze the calcium regulation during synaptic activities. In order to understand how the activity of these excitatory and inhibitory channels effect postsynaptic calcium, we run our simulations with four different protocols (see Figure 6).
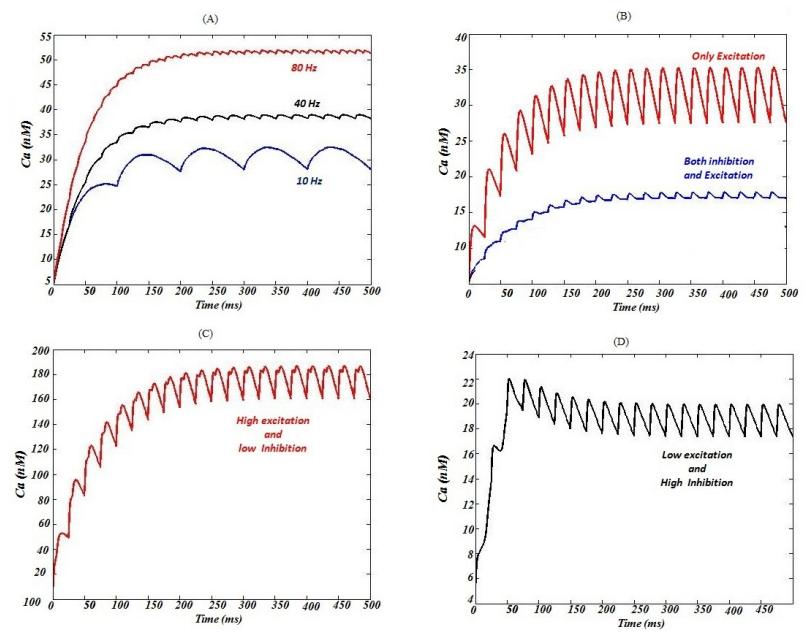

Figure 6. Plasticity of $\mathrm{Ca}^{2+}$ signals. Panel (A): Modulation of calcium signals in response to synaptic activity in spines. All of the four channels are contributing with equal activity level, blue line for the $\alpha$-range activity, black line for medium $\gamma$-range activity and red line for high $\gamma$-activity. Panel (B): Simulation for the cases of no excitation, no inhibition and also the coexistence state (when both are present). $\mathrm{Ca}^{2+}$ signals strongly inhibit in the presence of active GABAR's. Panel (C): Excitation is increased ten times by the the inhibition and an excessive increase in calcium signals can be seen. This can be referred as the case of seizure. Panel (D): Now the inhibition is increased by ten times than the excitation and as a result $\mathrm{Ca}^{2+}$ signals are strongly inhibited. This case lead to long term depression.

(A) Different level of activities, $\alpha$-range $10 \mathrm{~Hz}$, medium $\gamma$-range $40 \mathrm{~Hz}$ and high $\gamma$-range $80 \mathrm{~Hz}$. (B) Only excitation, only inhibition and the same level 
of excitation and inhibition. (C) Strong excitation and weak inhibition and finally (D) strong inhibition and weak excitation. The effect of these calcium signal modulations is depicted in Figure 6. The results from these simulations clearly explain the inhibitory effect on the cytosolic calcium modulation. For high excitation and weak inhibition, the amount of calcium is much higher as compare to the case of low excitation and high inhibition which directly affect the strength of synaptic connection as depicted in Figure 7A. Further, as the excitation level is increased, the calcium accumulation also raised and converge to some stable steady state value. The strength of the synaptic connection is also plotted against the accumulated amount of calcium in Figure 7B. Clearly the strength of the connection is directly proportional to the cytosolic calcium accumulation and this is in turn directly related to synaptic learning.
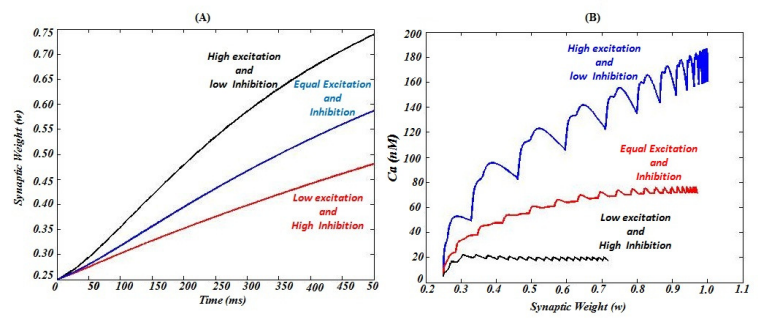

Figure 7. Synaptic learning rule. Level of the calcium concentration is strongly associated with the synaptic weights. Panel (A): Red line, inhibition is 10 times higher than excitation, so the synaptic connections are weak, black line, excitation is 10 times higher than inhibition, so a strong connection exist and finally the blue line, inhibition and excitation are at the same activity level, so as a result the synaptic connections are somehow in the medium range. Panel (B): The relationship of the $\mathrm{Ca}^{2+}$ modulation on the synaptic connection. More Calcium means strong connection.

\section{Discussion}

During the recent years, the concept of the brain plasticity has been emerged as the key player behind all types of brain functions; in particular, its cognition ability and injury induced recovery. Much of this plasticity is localized to the dendritic spines on post synaptic cell. Synaptic plasticity begins right away when an action potential arrive on presynaptic membrane. Spike transmission at the spike mediated chemical synapse is started with the invasion of an action potential from the neighboring neurons to the presynaptic terminal. As a result of this action potential, voltage dependent calcium channels open and lead to the rise of calcium concentration within the terminal. This causes synaptic vesicles to fuse near the cell membrane and release neurotransmitters into the synaptic cleft. While moving randomly in the cleft, some of the neurotransmitters bind with the specialized receptors on the postsynaptic membrane and make their way into the cytosol while many of them disperse. The diffusion of these transmitters into the cytosol not only change the postsynaptic potential but also alter the internal dynamic of the cell. 
In this paper, we introduced a physiologically realistic model to explain how the calcium signals can be modulated in the presence of inhibitory synapses. Recently it has been observed that active GABAergic synapses not only strongly effect the calcium signals but also have the ability to completely change the intracellular calcium dynamics in dendritic spines, see for instance $[4,5]$. But it is not clear how and upto what extent this modulation occurs. Probably it will not be an easy task because of the experimental constraints due to the complexity and small size of spine as well as the present indicators which are commonly used to measure the spine calcium activity can severely disrupt its dynamics. Our model has successfully described this phenomenon of synaptic modulation and encompasses both pre and postsynaptic dynamics which has not been previously documented in detail. Synaptic plasticity starts when randomly moving transmitter molecules bind with the postsynaptic cell. Pre and postsynaptic cells are coupled via chemical synapses and the probability of release of transmitter from presynaptic cell and to bind with the receptor on the postsynaptic cell has been elaborated. This probability increases with the rise in presynaptic activity. This means more activity on the presynaptic cell implies higher frequency of neurotransmitter release and a rapid opening and closing states on the binding sites of the postsynaptic membrane receptors. Our model suggests that probability of a state change (open to close and vice versa) of a gate on the postsynaptic cell remains in some particular range, but it will occur more frequently when excessive number of neurotransmitters are present in the cleft.

In the postsynaptic dynamics, we have discussed the contribution of excitatory and inhibitory currents in EPSP and IPSP. The excitatory synapses are strongly modulated when GABAergic receptors become active. Although, excitation show some resistance, but due to the strong inhibitory effect the cell spiking characteristics changes and a rapid hyperpolarization and a strong afterhyperpolarization can be observed. These postsynaptic potentials strongly modulate calcium signals inside the spine and a significant decline in the intracellular calcium level can be observed. Our model also supports the phenomenon that strong inhibition can lead to long term depression ( LTD) and the absence of the inhibition can cause excitotoxity, an extremely high level of synaptic activity due to seizure that causes a high influx of calcium. Our model also suggests that the level of intracellular calcium reduced by approximately half, when same amount of inhibition and excitation exist, as compared to the case when no inhibitory activity exists. Further in either case, the total intracellular calcium moves towards a steady state value as can be seen in Figure 6 .

Our model also elaborates the effect of these calcium signals on synaptic learning. The modulation in the calcium signals defines the coupling strength of the neurons. The strength of these coupling weights are considered to be responsible for the formation of memories and learning in the sense that a stronger connection will correspond to enhanced learning and a weaker connection may lead to depression. It can be seen that the synaptic weights are strong when we have high excitation and low inhibition and weak when we have low excitation and high inhibition. Further these weights also become weak in the situation when intracellular calcium concentration is low. 


\section{Concluding Remarks}

The size and complexity of neurons make it extremely hard for the experimentalist to understand their complete mechanism. Our model is a step toward the understanding of their complex nature. Although this model does not cover all the physiological aspects of the cell, it gives a direction to understand the functional role of inhibitory synapses on the postsynaptic calcium modulation. These effects have not been discussed through modeling before. Our model will also give a guideline to experimentalist to further test the hypothesis proposed here. A natural extension of this work is to investigate the modulation of calcium signals along the dendrite as well as to study a more detailed presynaptic dynamics whenever inhibitory synapses are active.

\section{References}

[1] A.G. Almonte, L.H. Qadri, F.A. Sultan, J.A. Watson, D.J. Mount and G. Rumbaugh. Protease-activated receptor-1 modulates hippocampal memory formation and synaptic plasticity. J. Neurochemistry, 124(1):109-122, 2013. http://dx.doi.org/10.1111/jnc.12075.

[2] B.L. Bloodgood and B.L. Sabatini. Neuronal activity regulates diffusion across the neck of dendritic spines. Science, 310(5749):866-869, 2005. http://dx.doi.org/10.1126/science.1114816.

[3] E.S. Bromberg-Martin, M. Matsumoto and O. Hikosaka. Control: Rewarding, aversive, and alerting. Neuron, 68(5):815-834, 2010. http://dx.doi.org/10.1016/j.neuron.2010.11.022.

[4] J.R. Chalifoux and A.G. Carter. GABAB receptors modulate NMDA receptor calcium signals in dendritic spines. Neuron, 66:101-113, 2010.

[5] C.Q. Chiu, G. Lur, T.M. Morse, N.T. Carnevale, G.C.R. Ellis-Davies and M.J. Higley. Compartmentalization of GABAergic inhibition by dendritic spines. Science, 340(6133):759-762, 2013. http://dx.doi.org/10.1126/science.1234274.

[6] S. Crook, M. Dur e Ahmad and S.M. Baer. A model of activity-dependent changes in dendritic spine density and spine structure. Math. Biosci. Engineering, 4(4):617-631, 2007. http://dx.doi.org/10.3934/mbe.2007.4.617.

[7] P. Dayan and L.F. Abbott. Theoretical Neuroscience: Computational and Mathematical Modeling of Neural Systems. MIT Press, 2001.

[8] A. Destexhe and T.J. Sejnowski. G protein activation kinetics and spillover of gamma-aminobutyric acid may account for differences between inhibitory responses in the hippocampus and thalamus. PNAS USA, 92:9515-9519, 1995. http://dx.doi.org/10.1073/pnas.92.21.9515.

[9] F.E. Dudek and T.P. Sutula. Epileptogenesis in the dentate gyrus: A critical perspective. Brain Research, 163:755-773, 2007.

[10] M. Dur e Ahmad, M. Imran and A. Gul. Calcium dynamics in dendritic spines: A link to structural plasticity. Math. Biosci., 230(2):55-66, 2011. http://dx.doi.org/10.1016/j.mbs.2011.01.002.

[11] N. Emptage, T.V. Bliss and A. Fine. Single synaptic events evoke NMDA receptor mediated release of calcium from internal stores in hippocampal dendritic spines. Neuron, 22:115-124, 1999.

http://dx.doi.org/10.1016/S0896-6273(00)80683-2. 
[12] Y. Hayashi and A.K. Majewska. Dendritic spine geometry: Functional implication and regulation. Neuron, pp. 529-532, 2005. http://dx.doi.org/10.1016/j.neuron.2005.05.006.

[13] H. Hering and M. Sheng. Dendritic spines: structure, dynamics and regulation. Nat. Rev. Neurosci., 12:880-888, 2001. http://dx.doi.org/10.1038/35104061.

[14] D. Holcman, E. Korkotian and M. Segal. Calcium dynamics in dendritic spines, modeling and experiments. Cell Calcium., 37:467-475, 2005. http://dx.doi.org/10.1016/j.ceca.2005.01.015.

[15] S. Honnuraiah and R. Narayanan. A calcium-dependent plasticity rule for HCN channels maintains activity homeostasis and stable synaptic learning. PLOS One, 8(2):1-17, 2013. http://dx.doi.org/10.1371/journal.pone.0055590.

[16] J.P. Johansen, C.K. Cain, L.E. Ostroff and J.E. LeDoux. Molecular mechanisms of fear learning and memory. Cell, 147(4):948, 2011.

http://dx.doi.org/10.1016/j.cell.2011.10.034.

[17] P.S. Keaser and W.G. Regehr. Molecular mechanism for synchronous, asynchronous and spontaneous neurotransmitter release. Ann. Rev. Physiol., 76:333363, 2014. http://dx.doi.org/10.1146/annurev-physiol-021113-170338.

[18] Y. Kovalchuk, J. Eilers, J. Lisman and A. Konnerth. NMDA receptor mediated subthreshold $\mathrm{Ca} 2+$ signals in spines of hippocampal neurons. Neurosci., 20:1791-1799, 2000.

[19] A. Kumar and M.R. Mehta. Frequency-dependent changes in NMDARdependent synaptic plasticity. Front. Comput. Neurosci., 5:1-15, 2011. http://dx.doi.org/10.3389/fncom.2011.00038.

[20] T. Kutsuwada, N. Kashiwabuchi, H. Mori, K. Sakimura, E. Kushiya, K. Araki, H. Meguro, H. Masaki, T. Kumanishi, M. Arakawa and M. Mishina. Molecular diversity of the NMDA receptor channel. Nature, 358:36-40, 1994. http://dx.doi.org/10.1038/358036a0.

[21] R. Lamprecht and J. LeDoux. Structure plasticity and memory. Nature Rev. Neurosci., 5:45-54, 2004. http://dx.doi.org/10.1038/nrn1301.

[22] D.M. Lovinger. Neurotransmitter roles in synaptic modulation, plasticity and learning in the dorsal striatum. Neuropharmacology, 58(7):951-961, 2010. http://dx.doi.org/10.1016/j.neuropharm.2010.01.008.

[23] Z.F. Mainen, R. Malinow and K. Svoboda. Synaptic calcium transients in single spines indicate that NMDA receptors are not saturated. Nature, 399(6732):151155, 1998.

[24] U.V. Nagerl, N. Eberhorn, S.B. Cambridge and T. Bonhoeffer. Bidirectional activity-dependent morphological plasticity and hippocampal neurons. Neuron, 44:759-767, 2004. http://dx.doi.org/10.1016/j.neuron.2004.11.016.

[25] E.A. Nimchinsky, B.L. Sabatini and K. Svoboda. Structure and function of dendritic spines. Ann. Rev. Physiol., 64:313-353, 2002. http://dx.doi.org/10.1146/annurev.physiol.64.081501.160008.

[26] T.S. Otis and I. Mody. Modulation of decay kinetics and frequency of GABAA receptor-mediated spontaneous inhibitory postsynaptic currents in hippocampal neurons. Neurosci., 49(1):13-32, 1992. http://dx.doi.org/10.1016/0306-4522(92)90073-B.

[27] P. Rhodes. The properties and implications of NMDA spikes in neocortical pyramidal cells. Neurosci., 26(25):6704-6715, 2006. http://dx.doi.org/10.1523/JNEUROSCI.3791-05.2006. 
[28] E.D. Schutter. Computational Modeling Methods for Neuroscientists. MIT Press, 2010.

[29] M. Segal, E. Korkotian and D.D. Murphy. Dendritic spine formation and pruning: Common cellular mechanism. Trends Neurosci., 23:53-57, 2000.

http://dx.doi.org/10.1016/S0166-2236(99)01499-X.

[30] D.W. Verzi, M.B. Rheuben and S.M. Baer. Impact of time-dependent changes in spine density and spine shape on the input-output properties of a dendritic branch: A computational study. J. Neurophysiol., 93:2073-2089, 2005. http://dx.doi.org/10.1152/jn.00373.2004.

[31] R. Yasuda, B.L. Sabatini and K. Svoboda. Plasticity of calcium channels in dendritic spines. Nature Neurosci., 6:948-955, 2003. http://dx.doi.org/10.1038/nn1112.

[32] D. Song Z. Wang and T.W. Berger. Contribution of NMDA receptor channels to the expression of LTP in the hoppicampal dentate gyrus. Hippocampus, 12:680688, 2002. http://dx.doi.org/10.1002/hipo.10104.

[33] A. Zador, C. Koch and T.H. Brown. Biophysical model of a Hebbiam synapse. PNAS, 87:6718-6722, 1990. http://dx.doi.org/10.1073/pnas.87.17.6718. 